\title{
Obesity indices comparison and its correlation with random blood sugar and blood pressure in adults in rural field practice area of a medical college
}

\author{
Harshal Gajanan Mendhe*, Hanumanth Narni, Satyanarayana Chowdary P., \\ Shashikanth M.
}

Department of Community Medicine, Dr PSIMS and RF, Chinoutapalli, AP, India

Received: 11 July 2016

Accepted: 06 August 2016

*Correspondence:

Dr. Harshal Gajanan Mendhe,

E-mail: drharshalmendhe@gmail.com

Copyright: ( ) the author(s), publisher and licensee Medip Academy. This is an open-access article distributed under the terms of the Creative Commons Attribution Non-Commercial License, which permits unrestricted non-commercial use, distribution, and reproduction in any medium, provided the original work is properly cited.

\begin{abstract}
Background: The prevalence of obesity has doubled in adults and children and tripled in adolescents over the past 2 decades. Body Mass Index (BMI) has gained international acceptance as a standard for recognition and classification of overweight and obesity. Measuring Waist Circumference (WC) alone is a simpler way of assessing for abdominal fat and has been shown by more recent research to be superior to WHR in determining health risks. This present study was conducted among the adults above 30 years adults in the rural field practice area of a medical college with the objectives to assess prevalence of obesity using BMI, WC, WHR and WHtR, to compare these indices and to correlate obesity indices with random blood sugar level and blood pressure.

Methods: It was a household survey in which 1150 participants were included as study subjects.

Results: It was observed that $16.8 \%$ study participants were obese as per International classification of BMI, 35\% study participants were obese as per BMI National classification and WHR, 26.7\% were obese as per WC classification and 66\% study participants were obese as per WHtR. In the present study, it was observed that none of the obesity indices was found to be correlated with Systolic B.P, Diastolic B.P and Random blood sugar level.

Conclusions: WHtR greater than 0.5 is a simple and effective indicator of centralized obesity. It measures obesity in those individuals which are healthy according to BMI and WC. One can use a simple instrument such as measuring tape to assess obesity as it measures WC, WHtR, and WHR instead of using heavy and non-calibrated weighing machine.
\end{abstract}

Keywords: BMI, WC, WHR, WHtR, RHTC

\section{INTRODUCTION}

Obesity has become the public health issue of the dayand for good reason. The data outline a dismal picture and a more foreboding future. The prevalence of obesity has doubled in adults and children and tripled in adolescents over the past 2 decades. ${ }^{1}$ Individuals from so called disadvantaged communities are not exempted from the epidemic. They are also at the risk of obesity and its complications. ${ }^{2}$ Hypertension is common in newly diagnosed type 2 Diabetes Mellitus and is associated with obesity. The association between hypertension and higher triglyceride and insulin levels may be secondary to obesity. An association between hypertension and cardiovascular complications is already apparent at the diagnosis of diabetes mellitus. ${ }^{3}$

A strong correlation has been established between a high BMI and the development of type 2 diabetes mellitus from a study of more than 7000 British men (mean follow up of 12 years). ${ }^{4}$ These observations were expected as obesity is known to induce insulin resistance due to decrease in insulin sensitive receptors as the weight increases. ${ }^{5}$ Positive correlations between BMI and 
glucose levels (random and fasting), body lipid level and blood pressure have been documented. ${ }^{6,7}$ A positive correlation is thought to exist between random blood glucose and obesity. However, racial factors seem to be important in the relationship between BMI and glucose intolerance because large studies such as Scottish study did not demonstrate a correlation between causal blood sugar and BMI. ${ }^{8}$

Body Mass Index (BMI) has gained international acceptance as a standard for recognition and classification of overweight and obesity. It is used by various health care professionals as an initial screen to identify individuals at risk for problems related to being overweight or underweight. Measuring Waist Circumference (WC) alone is a simpler way of assessing for abdominal fat and has been shown by more recent research to be superior to WHR in determing health risks. ${ }^{9}$

There has been some conflicting data regarding the relative merits of WHR verses $\mathrm{WC}$, but the current scientific consensus favors the use of WC. WHR is particularly inaccurate in women. ${ }^{10}$ World Health Organization has declared WHtR as the best indicator of cardiovascular risk. This index is age independent and sex independent. Cut off value of WHtR is 0.5. ${ }^{11}$ By comparison with BMI, WC, WHR and WHtR, WHtR more than 0.5 may be best indicator for undiagnosed type 2 diabetes mellitus and impaired fasting glucose. ${ }^{12}$

In young children, WHtR is not superior to WC or BMI in estimating Body Fat\%, nor is WHtR better correlated with cardio-metabolic risk factor than WC or BMI in overweight/ obese children. These data do not support the use of WHtR in young children. ${ }^{13}$ WHtR is a simple and practical anthropometric index to identify higher metabolic risk in normal and overweight Japanese men and women. ${ }^{14}$ So this present study was conducted among the adults above 30 years adults in the rural field practice area of a medical college with the objectives to assess prevalence of obesity using BMI, WC, WHR and WHtR, to compare these indices and to correlate obesity indices with random blood sugar level and blood pressure.

\section{METHODS}

It was a household survey conducted in the rural field practice area of a medical college among the adults above 30 years of age in the month of October 2015 to January 2016. In different settings, at different places in India, the prevalence of obesity was varied as per the results of different studies. Based on results from Pilot study, the prevalence of obesity was $25.8 \%$ among 150 adults above 30 years of age.

Taking $10 \%$ allowable error, the sample size will be 1150. The field practice area of RHTC covers 9 villages, out of them 3 villages were selected randomly using Lottery method. The population of these 3 villages was 9062 and the total households were1639. Systematic random sampling was used to select household from the villages as per the house hold data available at RHTC. Every $2^{\text {nd }}$ house hold was visited and the family members whose age was above 30 years were selected as study participants.

If the household was found locked during the survey, the right hand thumb rule was practiced. A total of 1150 participants were included as study subjects. The study participants were explained the purpose of the study. Written informed consent was obtained from all the participants and the study was approved by the Ethical Committee of a Medical College. The structured questionnaires were used to collect necessary information as per objectives of the study.

Preliminary information such as house no, religion, caste, ADHAR no. education, occupation and income was obtained. Each participant was asked about his family history of diabetes mellitus and hypertension and also his own past history of diabetes mellitus and hypertension with duration of treatment.

Weight and height were measured by trained nurses and interns. The measurement was carried out with a standard hospital weighing balance and height measure, in light clothing without shoes, jackets and heavy ornaments.

Table 1: Classification of adults according to BMI. ${ }^{11}$

\begin{tabular}{|llll|}
\hline \multicolumn{1}{r}{ Classification } & Global population & Asian population & Indian population \\
\hline Underweight & $<18.5$ & $<18.5$ & $<18.5$ \\
\hline Normal & $18.5-24.99$ & $18.5-22.99$ & $18.5-22.99$ \\
\hline Overweight & $25-29.99$ & $23-26.99$ & $23-24.99$ \\
\hline Obese & $\geq 30$ & $\geq 27$ & $\geq 25$ \\
\hline
\end{tabular}

Subjects were asked to stand erect on the scale with both arms at the sides. The weight was recorded to the nearest
$0.1 \mathrm{~kg}$ and height to the nearest $0.1 \mathrm{~cm}$. Height was measured as the distance from top of the head to the 
bottom of the feet (no shoes). Waist circumference was measured using measuring tape at the level between lower border of costal margin and iliac crest. Hip circumference was measured at the level of greater trochanter. Blood pressure was measured using Sphygmomanometer. The systolic and diastolic pressure was measured three times over a period of three minutes. Accordingly relevant data was collected, compiled and analyzed using SPSS- V19.

Table 2: Cut-off for WC. ${ }^{11}$

\begin{tabular}{|lll|}
\hline \multicolumn{2}{|c|}{ Population } & \\
& Male & $90 \mathrm{~cm}$ \\
\cline { 2 - 3 } & Female & $80 \mathrm{~cm}$ \\
\hline Global & Male & $102 \mathrm{~cm}$ \\
\cline { 2 - 3 } & Female & $88 \mathrm{~cm}$ \\
\hline
\end{tabular}

Waist to Height Ratio (WHtR) Cut off for WHtR-0.5. ${ }^{11}$ Cut off for Waist to Hip ratio (WHR) Male >1, Female > 0.85 . $^{11}$
Statistical tools applied were mean, percentages, Chisquare Test, Co-relation Coefficient, Logistic Regression, ROC curve. In the assessment of obesity, International classification of BMI was taken as gold standard and compared with the other obesity indices. ${ }^{15}$

\section{RESULTS}

$29.3 \%$ study participants were in the age group of 30 $40 \mathrm{yrs}, 25.7 \%$ were in the age group of $41-50$ years, $21.7 \%$ were in 51-60years and $23.3 \%$ were geriatric age group. $48.5 \%$ study participants were males and $51.5 \%$ were females. $19 \%$ study participants were known diabetic and $24 \%$ were hypertensive.

As per socio-economic status according to modified BGPrasad scale, $30 \%$ study participants were belonged to middle socio-economic status group, $25 \%$ belonged to upper middle, $21 \%$ belonged to lower middle, $17 \%$ lower and only $7 \%$ belonged to upper class.

Table 3: Comparison of different obesity indices with BMI international.

\begin{tabular}{|llllllll|}
\hline \multirow{2}{*}{ Measurement } & Obese & \multicolumn{9}{c|}{ Comparison with International classification of BMI } \\
& $\mathbf{n}$ & $\mathbf{\%}$ & Sensitivity & Specificity & Area under ROC & LL & UL \\
\hline BMI International & 193 & 16.8 & - & - & - & - & - \\
\hline BMI National & 400 & 34.8 & 100 & 78.4 & 0.89 & 0.87 & 0.91 \\
\hline WC & 307 & 26.7 & 94.8 & 87 & 0.91 & 0.89 & 0.93 \\
\hline WC by Height & 762 & 66.3 & 99.5 & 40.4 & 0.7 & 0.67 & 0.73 \\
\hline WC by Hip & 401 & 34.9 & 94.8 & 77.2 & 0.86 & 0.84 & 0.89 \\
\hline
\end{tabular}

In the present study, it was observed that $16.8 \%$ study participants were obese as per International classification of BMI, 35\% study participants were obese as per BMI National classification and WHR, $26.7 \%$ were obese as per WC classification and $66 \%$ study participants were obese as per WHtR.

Maximum prevalence was found in cases of WHtR which measures abdominal obesity. In assessment of obesity, International classification of BMI was taken as Gold standard and compared with other obesity indices. It was found that $100 \%$ sensitivity was found in case of BMI national and $99.5 \%$ sensitivity was observed in case of WHtR.

But in case of WHtR, it lacks the specificity (40.4\%). After comparison with Gold standard, area under ROC was less in case of WHtR.

In assessment of obesity by WHtR, $58 \%$ overweight and $63 \%$ normal individuals were also detected as obese. The relation between WHtR and categories of BMI International was found to be statistically significant.
Table 4: Correlation of Obesity indices with BP and RBS.

\begin{tabular}{|lll|}
\hline Relation with BMI & $\mathbf{r}$ & $\mathbf{P}$ \\
\hline SBP & 0.08 & 0.01 \\
\hline DBP & 0.04 & 0.14 \\
\hline RBS & 0.03 & 0.3 \\
\hline Relation with WC & $\mathbf{r}$ & $\mathbf{P}$ \\
\hline SBP & 0.08 & 0.01 \\
\hline DBP & 0.06 & 0.04 \\
\hline RBS & 0.02 & 0.60 \\
\hline Relation with WHR & $\mathbf{r}$ & $\mathbf{P}$ \\
\hline SBP & 0.04 & 0.15 \\
\hline DBP & 0.03 & 0.40 \\
\hline RBS & 0.001 & 0.97 \\
\hline Relation with WHtR & $\mathbf{r}$ & $\mathbf{P}$ \\
\hline SBP & 0.06 & 0.03 \\
\hline DBP & 0.04 & 0.15 \\
\hline RBS & 0.02 & 0.53 \\
\hline
\end{tabular}


In the assessment of obesity by WHR, 32\% overweight and $21 \%$ normal individual were also deemed as obese. The relation between WHR and categories of BMI International was found to be statistically significant. In assessment of obesity by WC, 53\% overweight and $2 \%$ normal individuals were also deemed as obese. The relation between WC and categories of BMI International was found to be statistically significant. In the present study, it was observed that obesity was more common among females (19\%) as compared to males (14\%) but was found to be statistically significant.

In the present study, it was observed that none of the obesity indices was found to be correlated with Systolic B.P, Diastolic B.P and Random blood sugar level. But association between Systolic B.P with obesity indices such as International BMI, WC and WHR was found to be statistical significant.

Table 5: Association between BMI International categories with SBP.

\begin{tabular}{|c|c|c|c|c|c|}
\hline \multirow[t]{2}{*}{ BMI Internation } & & SBP & \multirow[t]{2}{*}{ Total } & \multirow[t]{2}{*}{$x^{2}$} & \multirow[t]{2}{*}{ P-value } \\
\hline & $\leq 140 \mathrm{mmHg}$ & $>140 \mathrm{mmHg}$ & & & \\
\hline \multirow[t]{2}{*}{ Under weight } & 27 & 8 & 35 & \multirow[t]{10}{*}{10.64} & 0.014 \\
\hline & $77.14 \%$ & $22.86 \%$ & $100.00 \%$ & & \\
\hline \multirow[t]{2}{*}{ Normal } & 524 & 191 & 715 & & \\
\hline & $73.30 \%$ & $26.70 \%$ & $100.00 \%$ & & \\
\hline \multirow[t]{2}{*}{ Over weight } & 146 & 61 & 207 & & \\
\hline & $70.50 \%$ & $29.50 \%$ & $100.00 \%$ & & \\
\hline \multirow[t]{2}{*}{ Obese } & 119 & 74 & 193 & & \\
\hline & $61.70 \%$ & $38.30 \%$ & $100.00 \%$ & & \\
\hline \multirow[t]{2}{*}{ Total } & 816 & 334 & 1150 & & \\
\hline & $70.96 \%$ & $29.04 \%$ & $100.00 \%$ & & \\
\hline
\end{tabular}

The association between categories of BMI International and systolic BP (hypertensive/ non hypertensive's) was found to be statistically significant as $38 \%$ obese and $30 \%$ overweight were found hypertensive. Same was found in case of diastolic BP and categories of BMI International as $46 \%$ obese and $37 \%$ overweight were hypertensive.

But the relation between categories of BMI International and sugar level $<140 \mathrm{mg} \%$ and $>140 \mathrm{mg} \%$ was found to be statistically non-significant. Obesity was found to be associated with patients having only hypertension, with patients having only diabetes but was more associated with patients having both hypertension and diabetes. This association was not found to be statistically significant.

\section{DISCUSSION}

This study was conducted among the adults above 30 years adults in the rural field practice area of a medical college with the objectives to assess prevalence of obesity using BMI, WC, WHR and WHtR, to compare these indices and to correlate obesity indices with random blood sugar level and blood pressure.

In the present study, it was observed that $16.8 \%$ study participants were obese as per International classification of BMI, 35\% study participants were obese as per BMI National classification and WHR, 26.7\% were obese as per WC classification and $66 \%$ study participants were obese as per WHtR. Maximum prevalence was found in cases of WHtR which measures abdominal obesity. A study done by Sachin $\mathrm{H}$ et al in aged 20 and over years old males living in 11 towns in Kayseri, Turkey, according to BMI, overweight and obesity prevalence were $39.6 \%$ and $16.9 \%$, respectively. Obesity prevalence was $29.9 \%$ (50-59 years) according to WHR, 50.4\% (6069 years) according to Waist Circumference (WC) and $24.8 \%$ (40-49 years) according to WHtR. ${ }^{16}$

In 1994, ICMR task force carried out a study involving more than 5000 individuals (3050 urban residents and 2487 rural residents) demonstrated $43 \%$ prevalence of overweight in urban Delhi and $12 \%$ in rural Haryana. ${ }^{17}$ Prevalence in the rural areas of Tamil Nadu rose sharply from $2 \%$ in 1989 to $17 \%$ in $2003 .{ }^{18}$ Kutty VR carried out a study in rural Kerala during 1991 using criteria of BMI $>27$, and the prevalence was found to be $5.8 \%{ }^{19}$

Later studies in Kerala reported $49 \%$ prevalence of overweight among 30-64 age group in 1998 and $41 \%$ among 40-60 age group during 2000. ${ }^{20,21}$ ICMR Task force study (1994) reported a higher prevalence in urban Delhi (31\%) and a lower prevalence in rural Haryana $(8 \%$ ) using the criteria (WC $>94 \mathrm{~cm}$ among men and $>88 \mathrm{~cm}$ among women). ${ }^{17}$ The Sentinel surveillance on CVD in Indian industrial population (2001-03), using modified ATP III criteria for Asian (WC $>90 \mathrm{~cm}$ among men and $>80 \mathrm{~cm}$ among women) reported an overall prevalence of abdominal obesity at $32 \%$. Dibrugarh in 
Assam had the lowest prevalence (0.7\%) while Hyderabad in Andhra Pradesh had the highest (52\%). ${ }^{22}$ The ICMR Task force project reported $65 \%$ prevalence of high waist hip ratio in urban Delhi and 52\% in rural Haryana in 1994 using the criteria ( WHR: M->0.9, F$>0.8)^{17}$

A study done by Wen Cheng Li et.al among Taiwanese adults, it was concluded that a WHtR greater than 0.5 is a simple and yet effective indicator of centralized obesity and associated cardio-metabolic risk, even among individuals deemed healthy according to BMI and WC.

In the same study, it was observed that the area under curve of WHtR was significantly greater than that of BMI or WC in the prediction of high total cholesterol, high triglyceride and low HDL-cholesterol. ${ }^{23}$ In the present study also, it was found that individuals who were normal and overweight according to BMI, found to be obese according to WHtR greater than 0.5 .

A strong correlation has been established between a high BMI and the development of type 2 diabetes mellitus from a study of more than 7000 British men (mean follow up of 12 years). ${ }^{4}$ In the present study, it was observed that none of the obesity indices was found to be correlated with Systolic B.P, Diastolic B.P and Random blood sugar level. But association between Systolic B.P and International BMI, WC and WHR was found to be statistical significant.

Positive correlations between BMI and glucose levels (random and fasting), body lipid level and blood pressure have been documented. ${ }^{6,7} \mathrm{~A}$ positive correlation is thought to exist between random blood glucose and obesity. However, racial factors seem to be important in the relationship between BMI and glucose intolerance because large studies such as Scottish study did not demonstrate a correlation between causal blood sugar and BMI. ${ }^{8}$

"Central obesity" indicated by an increased WHR, has been positively correlated with high blood pressure in several populations. ${ }^{24}$ In the present study, it was observed that none of the obesity indices was found to be correlated with Systolic B.P, Diastolic B.P and Random blood sugar level. But association between Systolic B.P and International BMI, WC and WHR was found to be statistical significant.

The relation between categories of BMI International and systolic BP (hypertensive/ non hypertensive's) was found to be statistically significant as $38 \%$ obese and $30 \%$ overweight were found hypertensive.

Strength of the study was that larger sample size was employed and standard procedure was used to classify study participants as per guidelines i.e. cut off values. Limitation was that causal association between obesity and diabetes and hypertension could not be determined.

\section{CONCLUSION}

WHtR greater than 0.5 is a simple and effective indicator of centralized obesity. It measures obesity in those individuals which are healthy according to BMI and WC. One can use a simple instrument such as measuring tape to assess obesity as it measures WC, WHtR, and WHR instead of using heavy and non-calibrated weighing machine.

\section{ACKNOWLEDGEMENTS}

Authors would like to thank the management of Dr PSIMS \& RF medical college, Chinnaoutapalli, for their financial and material support, HOD of community medicine for his valuable guidance in accomplishment of house hold survey.

Funding: Management of Dr PSIMS and RF medical college, Chinnaoutapalli

Conflict of interest: None declared

Ethical approval: The study was approved by the Institutional Ethics Committee

\section{REFERENCES}

1. Bassett MT, Perl S. Obesity: The Public Health Challenge of Our Time. Am J Public Health. 2004;94(9):1477.

2. Haslam DW, James WP. Obesity. Lancet. 2005;366:1197-209.

3. Turner RC, Holman RR, Matthews. Prevalence of hypertension in newly presenting Type-II Diabetic patients and the association with risk factors for cardiovascular and diabetic complications. J Hypertens. 1993;11(3):309-17.

4. Kahn CR. Banting Lecture. Insulin action, diabetogenes and the causes of type 2 diabetes. Diabetes. 1994;43:1066-84.

5. Sue RW, Sara LA. Nutrition and Diet Therapy, 7th (ed), Mosby, USA. 1993.

6. Pucarin-Cvetković J, Mustajbegović J, Doko Jelinić J, Senta A, Nola IA, Ivanković D, et al. Body mass index and nutrition as determinants of health and disease in population of Croatian Adriatic islands. Croat Med J. 2006;47:619-26.

7. Turcato E, Bosello O, Di Francesco V. Waist circumference and abdominal sagittal diameter as surrogates of body fat distribution in the elderly; their relation with cardiovascular risk factors. Int $\mathbf{J}$ Obese Relat Metab Disord. 2000;24:1005-10.

8. Janghorbani M, Hedley AJ, Jones RB. Is the association between glucose level and "all causes" and cardiovascular mortality risk dependant on Body mass index? Med J JRI. 1991;6:205-12.

9. Kuczmarski RJ, Flegal KM. Criteria for definition of overweight in transition: Background and recommendations for the United States. Am. J. Clin. Nutr. 2000;72:1074-81. 
10. Rankinen T, Kim S, Perusse L, Desprs H, Gouchard C. The prediction of abdominal visceral fat level from body composition and anthropometry: ROC analysis. Int. J. Obesity Related Metabolic Res. 1999;23:801-9.

11. Jain V. Review of Preventive and Social Medicine including Biostatistics. JAYPEE BROTHERS MEDICAL PUBLISHER, New Delhi. 6th edition, 2014;290-2.

12. Xu Z, Qi X, Dahl AK, Xu W. Waist to height ratio is the best indicator for undiagnosed type-II diabetes. Diabet Med. 2013;30(6):e201-7.

13. Sijtsma A, Bocca G, Abee CL. Waist to height ratio, Waist circumference and BMI as indicators of percentage of fat mass and cardio metabolic risk factors in children aged 3-7 years. Journal of Clinical Nutrition. 2014;33(2):311-5.

14. Hsieh SD. Waist to height ratio, a simple and practical index for assessing central fat distribution and metabolic risk in Japanese men and women. Int J Obes Relat Metab Disord. 2003.

15. Odenigbo UM, Odenigbo UC, Oguejiofor OC, Adogu POU. Relationship of Waist Circumference, Waist Hip Ratio \& Body Mass Index as Predictors of Obesity in Adult Nigerians. Pakistan Journal of Nutrition. 2011;10(1):15-8.

16. Sachin H, Cicek B, Yilmaz M, Ongan D, Inanc N. Obesity prevalence, waist to height ratio and associated factors in adult Turkish males. Obes Res Clin Pract. 2011;5(1):e29-35.

17. ICMR Task force project on Collaborative study of coronary Heart Study. Available at: http://www.searo.who.int/ india/ topics/ cardiovascular_diseases/ NCD_Resources_ National_CVD_database-Final_Report.pdf?ua $=1$
18. Ramachandran A, Snehalatha C, Baskar ADS, Mary S, Kumar CKS, Selvam S, et al. Temporal changes in prevalence of diabetes and impaired glucose tolerance associated with lifestyle transition occurring in the rural population in India. Diabetologia. 2004;47:860-5.

19. Kutty VR, Balakrishnan KG, Jayasree AK, Thomas J. Prevalence of coronary heart disease in the rural population of Thiruvananthapuram district, Kerala, India. Int J Cardiol. 1993;39(1):59-70.

20. Kutty VR, Soman CR, Joseph A, Kumar KV, Pisharody R. Random capillary blood sugar and coronary risk factors in a south Kerala population. J Cardiovasc Risk. 2002;9(6):361-7.

21. Zachariah MG, Thankappan KR, Alex SC, Sarma PS, Vasan RS. Prevalence, correlates, awareness, treatment, and control of hypertension in a middleaged urban population in Kerala. Indian Heart $\mathbf{J}$. 2003;55(3):245-51.

22. WHO-Project on sentinel surveillance of Indian Industrial population.

23. Wen-Cheng Li, I-Chuan Chen, Yu-Che Chang. Waist to height ratio, waist circumference and BMI as indices of Cardiometabolic risk among 36,642 Taiwanese adults. Eur J Nutr. 2013;52(1):57-65.

24. Park K. Text book of Preventive and Social Medicine. Bhanot publishers, Jabalpur. 21 st Ed. 347.

Cite this article as: Mendhe HG, Narni H, Chowdary SP, Shashikanth M. Obesity indices comparison and its correlation with random blood sugar and blood pressure in adults in rural field practice area of a medical college. Int J Community Med Public Health 2016;3:2555-60. 